\title{
Multiresolution-based registration of a volume to a set of its projections
}

\author{
Slavica Jonić, Philippe Thévenaz, Michael Unser \\ Swiss Federal Institute of Technology Lausanne, CH-1015 Lausanne VD, Switzerland
}

\begin{abstract}
We have developed an algorithm for the rigid-body registration of a 3D CT to a set of C-arm images by matching them to computed cone-beam projections of the CT (DRRs). We precomputed rescaled versions (pyramid) of the $\mathrm{CT}$ volume and of the $\mathrm{C}$-arm images. We perform the registration of the CT to the C-arm images starting from their coarsest resolution until we reach some finer resolution that offers a good compromise between time and accuracy. To achieve precision, we use a cubic-spline data model to compute the data pyramids, the DRRs, and the gradient and the Hessian of the cost function. We validate our algorithm on a 3D CT and on C-arm images of a cadaver spine using fiducial markers. When registering the CT to two C-arm images, our algorithm operates safely if the angle between the two image planes is larger than $10^{\circ}$. It achieves an accuracy of approximately $2.0 \pm 1.0 \mathrm{~mm}$.
\end{abstract}

Keywords: 3D/2D image registration, intensity-based registration, DRR, B-splines, multiresolution

\section{INTRODUCTION}

We propose an algorithm to register a volume of unknown pose to a set of reference projections with known poses. Our algorithm is similar to several published ones. ${ }^{1-7}$ It relies on computing projections of the volume and on matching them to the reference projections. It iteratively optimizes a mean-squares cost function and uses a Marquardt-Levenberg algorithm for acceleration. We refer to the computed projections as Digitally Reconstructed Radiographs (DRRs). We obtain them by integrating the volume along simulated X-rays. The potential applications of our algorithm are in computer-assisted orthopedic surgery, ${ }^{8}$ where it is useful to integrate a high-resolution preoperative 3D CT data into the intraoperative procedure by using intraoperative C-arm images of the patient. We have refined our previous registration algorithm ${ }^{9}$ in two ways. Firstly, to compute DRRs, our algorithm can now handle the cone-beam geometry in addition to the parallel-beam geometry. Secondly, we compare several multiresolution approaches and we implement one that gives the best trade-off between the registration accuracy and the registration time.

To compute the gradient of the cost function exactly, we interpolate the volume using a cubic-spline data model. ${ }^{10}$ We take advantage of our shearing $\operatorname{method}^{9}$ to perform a fast computation of a DRR. This method is related to shear-warp factorization, ${ }^{7}$ but, contrary to, ${ }^{7}$ does not require an intermediate DRR to be computed.

In this paper, we validate our algorithm on a 3D CT and on C-arm images of a cadaver spine. We evaluate our estimation of the pose of the CT in the reference coordinate system against a ground-truth pose that is known a priori by way of fiducial markers implanted in the specimen before that image data were acquired.

\section{REGISTRATION ALGORITHM}

\subsection{Multiresolution}

Given a CT volume and a set of C-arm images, we precompute their cubic-spline $L_{2}$ pyramids. A cubic degree offers a compromise between interpolation quality and computation time. We estimate the pose of the CT in the reference coordinate system by refining an initial CT pose. We register the volume to the images starting from their coarsest resolution. We use the final estimate obtained at some pyramid level to resume the registration at the next finer one. We repeat the registration procedure at even finer resolutions until we reach a resolution that provides a good compromise between registration accuracy and registration time.

Correspondence: slavica.jonic@epfl.ch, http://bigwww.epfl.ch/ 


\subsection{Cone-beam projection}

Given a pose $\boldsymbol{\mu}$ of the volume $f$, the pose of the projecting plane, and the coordinate $\mathbf{r}$ of the illumination source in the reference coordinate system, we compute the cone-beam DRR $p \boldsymbol{\mu}$ by casting simulated X-rays through the volume, from the grid points in the plane towards the source.

Let $\mathbf{u}_{\mathbf{i}}$ be the unit vector on the ray from the source to the point with the index $\mathbf{i}=\left(i_{1}, i_{2}, 0,1\right)$ in the image plane. Let $\mathbf{B}_{\boldsymbol{\mu}}$ be a rigid-body transformation from the system of volume indexes to the reference coordinate system, and let $\mathbf{B}_{\boldsymbol{\mu}}^{-\mathbf{1}}$ be its inverse transformation. We propose to write the DRR as follows:

$$
p_{\boldsymbol{\mu}}(\mathbf{i})=\int_{e_{\mathbf{i}}}^{l_{\mathbf{i}}} f\left(\mathbf{B}_{\boldsymbol{\mu}}^{-1}\left(\mathbf{r}+t \mathbf{u}_{\mathbf{i}}\right)\right) \mathrm{d} t .
$$

Direct implementation of Equation (1) would require a 3D interpolation of the volume $f$. We perform a fast computation of a DRR by replacing the 3D interpolation by a $2 \mathrm{D}$ interpolation. This method, that we call shearing, ${ }^{9}$ takes advantage of a change of the integration variable $t$ in Equation (1), and of an approximation of the integral by a discrete sum. We adapt the size of the sampling step along $t$ so that only samples of the volume with one integer and two real coordinates take part in the sum.

\section{DATA}

A cadaver spine specimen* was frozen so that it can be treated as a rigid body. Five fiducial markers were implanted on it. One was placed in the L5, two in the L4, and two in the L3 vertebra. The specimen was CT-scanned with 72 slices of size 512 x 512 pixels each (Fig. 1). The intra-slice pixel size was $0.36 \times 0.36 \mathrm{~mm}$, and the inter-slice thickness was $2.5 \mathrm{~mm}$. The tilt angle was zero.
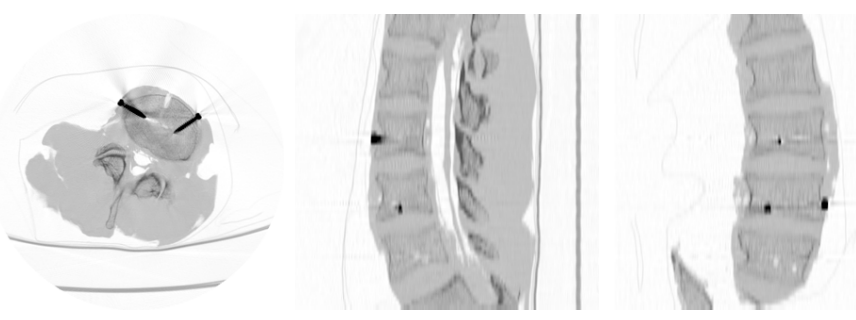

Figure 1. Transversal (left), sagital (center), and frontal (right) intersections of the CT with visible fiducial markers.
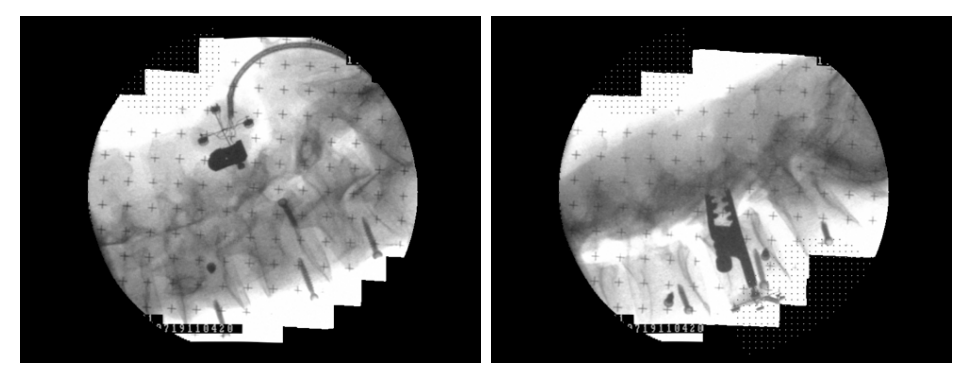

Figure 2. Two C-arm images with a visible dynamic reference base.

A Dynamic Reference Base (DRB) was attached on the specimen before shooting the C-arm images in a way that it projects onto the images. The presence of the DRB in C-arm images challenges our registration algorithm because the DRB is not present in the CT data. This situation would be typical in a clinical setting. C-arm

\footnotetext{
${ }^{*}$ Courtesy of the M.E. Müller Institute for Biomechanics, Bern, Switzerland.
} 
images were captured with seven different poses, and the fiducial markers were digitized in the DRB coordinate system by using a pointer. C-arm images of size 768 x 576 pixels, with pixel size $0.36 \times 0.36$ mm, were acquired (Fig. 2).

\section{VALIDATION}

\subsection{Ground-truth registration}

Given a list of the ground-truth DRB coordinates of the fiducial markers $\left(\mathbf{v}_{i}, i=1, \ldots, 5\right)$, and a list of their ground-truth 3D CT indexes $\left(\mathbf{k}_{i}, i=1, \ldots, 5\right)$, we estimate the ground-truth transformation $\mathbf{B}$ that transforms the system of $\mathrm{CT}$ indexes into the DRB coordinate system by minimizing $\chi^{2}=\frac{1}{5} \sum_{i=1}^{5}\left\|\mathbf{v}_{i}-\mathbf{B} \mathbf{k}_{i}\right\|^{2}$.

If the specimen was rigid and if $\mathbf{v}_{i}$ and $\mathbf{k}_{i}$ were obtained with perfect precision, it would be possible to get $\chi=0 \mathrm{~mm}$. However, we observe $\chi=0.76 \mathrm{~mm}$. This non-zero $\chi$ indicates that either the specimen was not rigid, or that the markers were digitized with some error, or that the ground-truth CT indexes of the markers were inexact. Independent analysis has shown that the digitization error is about $0.5 \mathrm{~mm}$.

\subsection{Misregistration}

In order to define a measure of misregistration, we transform every $3 \mathrm{D}$ CT index $\mathbf{k}$ into a DRB coordinate by using two transformations: the ground-truth transformation $\mathbf{B}$ and the transformation $\mathbf{B} \boldsymbol{\mu}$ that we estimate by performing image registration. We define the misregistration as the average of the norm of the difference between the two DRB coordinates over all the CT indexes $(\operatorname{card}(f)=512 \times 512 \times 72$ indexes), that is,

$$
M=\frac{1}{\operatorname{card}(f)} \sum_{\mathbf{k} \in f}\|(\mathbf{B}-\mathbf{B} \boldsymbol{\mu}) \mathbf{k}\| .
$$

\subsection{Experiments}

We want to determine the performance of our algorithm when registering the CT volume to a pair of C-arm images. We mask the fiducial markers in each of the two imaging modalities so that they do not influence the registration. Our algorithm proceeds by refining an initial condition. On purpose, we set this initial condition $\boldsymbol{\mu}_{0}$ such that $\mathbf{B} \neq \mathbf{B} \boldsymbol{\mu}_{0}$, which leads to a large initial misregistration $M_{0}$. A successful registration will lead to a final $M<<M_{0}$.

One of the elements that determine the working range of our algorithm is given in terms of the angle between the two involved image planes. It operates safely if the angle between the two image planes is larger than $10^{\circ}$. From the set of 21 image pairs $\left(\frac{7 !}{2 ! 5 !}=21\right)$, we perform the experiments on 19 pairs that satisfy the working conditions of our algorithm.

We use four-level CT and C-arm image pyramids. These pyramids are dyadic, but we do not change the number of CT slices while performing the data reduction. We perform the registration at the two coarsest pyramid levels only, since this strategy gives a good trade-off between accuracy and time. If we perform additional processing at the two finest data resolutions, we do not gain more than $0.2 \mathrm{~mm}$ accuracy. This does not justify the huge additional registration time. We did try to coarsen the data further, for example, by computing five-level pyramids, or by reducing the number of CT slices. However, such pyramids did not improve the performance of our registration algorithm since it had either failures at the coarser data resolutions or did not perform remarkably better at the finer data resolutions.

\subsection{Results}

We show in Table 1 the mean and the standard deviation of the misregistration $M$ over all 19 experiments (Sec. 4.3) for an initial fiducial-marker misregistration of approximately $9.02 \mathrm{~mm}$.

We were able to align the CT and C-arm images with a misregistration $M=1.79 \pm 0.69$ mm. The non-zero misregistration can be explained by the fact that the specimen was not perfectly rigid, that some errors were made when digitizing the fiducial markers in the DRB coordinate system, and that some more errors were made when determining the ground-truth CT indexes of the markers. At this time, it is very difficult to disentangle 
these effects to determine with more precision which fraction of the misregistration our algorithm is responsible for.

As far as the speed is concerned, we performed the experiments on a Power Mac G4, 733MHz, where the computation of volume and image pyramids took approximately $20 \mathrm{sec}$ and $6 \mathrm{sec}$, respectively. We achieved the registration in $5 \mathrm{~min}$ (Table 1 ).

Table 1. Results of the registration at the two coarsest data resolutions.

\begin{tabular}{|c|c|c|c|c|c|c|}
\hline Level & CT Size & C-Arm Image Size & \multicolumn{2}{|c|}{ Misregistration [mm] } & \multicolumn{2}{|c|}{ Time [sec] } \\
\cline { 4 - 6 } & {$[\mathrm{pxls}]$} & & Mean & Standard Deviation & Mean & Standard Deviation \\
\hline 3 & $64 \times 64 \times 72$ & $96 \times 72$ & 2.10 & 0.69 & 54.1 & 7.3 \\
\hline 2 & $128 \times 128 \times 72$ & $192 \times 144$ & 1.79 & 0.69 & 152.3 & 79.3 \\
\hline 1 & $256 \times 256 \times 72$ & $384 \times 288$ & ignored & ignored & ignored & ignored \\
\hline 0 & $512 \times 512 \times 72$ & $768 \times 576$ & ignored & ignored & ignored & ignored \\
\hline
\end{tabular}

\section{ACKNOWLEDGMENTS}

This work was supported in part by grant 02-U61 from the AO ASIF Foundation, Switzerland. We thank Dr Guoyan Zheng, Dr Richard Bächler, and Dr Lutz-Peter Nolte from the M.E. Müller Institute for Biomechanics, Bern, Switzerland, for providing us with the data.

\section{REFERENCES}

1. L. G. Brown and T. Boult, "Registration of planar film radiographs with computed tomography," in $I E E E$ Proc. MMBIA'96, pp. 42-51, 1996.

2. D. LaRose, J. Bayouth, and T. Kanade, "Transgraph: Interactive intensity-based 2D/3D registration of X-ray and CT data," in Proc. SPIE. Medical Imaging 2000: Image Processing, K. M. Hanson, ed., 3979, pp. 385-396, 2000 .

3. L. Lemieux, R. Jagoe, D. Fish, N. Kitchen, and D. Thomas, "A patient-to-computed-tomography image registration method based on digitally reconstructed radiographs," Med. Phys. 21, pp. 1749-1760, 1994.

4. G. Penney, J. Weese, J. Little, P.Desmedt, D. Hill, and D. Hawkes, "A comparison of similarity measures for use in 2D-3D medical image registration," IEEE Trans. Med. Imaging 17, pp. 586-595, 1998.

5. D. Sarrut and S. Clippe, "Geometrical transformation approximation for 2D/3D intensity-based registration of portal images and CT scan," in Proc. MICCAI 2001, pp. 532-540, 2001.

6. J. Weese, G. Penney, P. Desmedt, T. Buzug, D. Hill, and D. Hawkes, "Voxel-based 2-D/3-D registration of fluoroscopy images and CT scans for image-guided surgery," IEEE Trans. Inform. Technol. Biomedicine 1, pp. 284-293, 1997.

7. J. Weese, R. Göcke, G. Penney, P. Desmedt, T. Buzug, and H. Schumann, "Fast voxel-based 2D/3D registration algorithm using a volume rendering method based on the shear-warp factorization," in Proc. SPIE'99 Image Processing, 3661, pp. 802-810, 1999.

8. E. Bainville, I. Bricault, P. Cinquin, and S. Lavallée, "Concepts and methods of registration for computerintegrated surgery," in Computer Assisted Orthopedic Surgery (CAOS), L. Nolte and R. Ganz, eds., pp. 1534, Hogrefe \& Huber Publishers, Seattle-Toronto-Bern-Göttingen, 1999.

9. S. Jonić, P. Thévenaz, and M. Unser, "Multiresolution spline-based 3D/2D registration of CT volume and C-arm images for computer-assisted surgery," in Proc. SPIE. Medical Imaging 2001: Image Processing, M. Sonka and K. M. Hanson, eds., 4322, pp. 1101-1109, 2001.

10. M. Unser, "Splines: A perfect fit for signal and image processing," IEEE Signal Processing Magazine 16, pp. 22-38, 1999. 